\title{
Dynamique d'établissement d'un peuplement bi-étagé de bouleau blanc et de sapin baumier en zone boréale
}

\author{
par Louis Bélanger, Daniel Allard ${ }^{1}$ et Philippe Meek
}

The age structure of a boreal two-storied stand formed by an upperstory of white birch (Betula papyrifera Marsh.) with an understory of balsam fir (Abies balsamea (L.) Mill.) was analysed. The establishment of this 50 -year stand followed a burn that ran through a cut-over area. The objective was to verify if the establishment of the fir understory followed the traditional succession model or the initial floristic composition model. The age structure analysis indicated an immediate and rapid establishment of white birch during a period of 6 years after the fire. Establishment of fir, however, was initiated only 16 years after the fire. Peak establishment was 33 years after the fire. Fir regeneration followed a cycle. The regeneration pattern was characterized by the absence of fir establishment the first 16 years after the fire, followed by sporadic fir regeneration the next 12 years, then by a 13-year period of abundant regeneration after which there was a complete halt to fir regeneration. So, in this case, the successional model was more fit to describe the dynamics of balsam fir. The absence of fir regeneration during the initial period after the fire could be explained by the absence of the nearby fir seed sources due to harvesting. However, the total absence of fir establishment during the last period is more difficult to explain. Seedbed evolution since the fire could possibly be in cause. This and other studies indicate that in the boreal balsam fir-white birch ecoclimatic domain vegetation dynamic after fire does not limit itself to one pathway. Stand regeneration after fire is significantly affected by local conditions.
La structure d'âge d'un peuplement boréal bi-étagé constitué d'une strate arborescente de bouleau blanc (Betula papyrifera Marsh.) et d'un sous-étage arbustif de sapin baumier (Abies balsamea (L.) Mill.) a été étudiée. Ce peuplement a pour origine un feu datant d'une cinquantaine d'années survenu après la récolte des sapinières dans ce secteur. Le but de l'étude était de vérifier si la dynamique d'établissement du sapin suivait l'hypothèse classique de la succession ou le modèle de la composition floristique initiale. Les structures d'âge obtenues indiquent que le bouleau s'est établi au cours des six premières années qui ont suivi le feu. Le sapin, par contre, a pris 16 ans avant de commencer à s'établir. Son pic d'établissement se situe 33 ans après le feu. L'installation du sapin a suivi un patron qui se rapproche d'une normale. Ce patron est caractérisé par l'absence d'établissement de sapin les premiers 16 ans, une phase d'installation sporadique les 12 années suivantes, une phase d'installation abondante d'environ 13 ans suivie d'une dernière phase où l'établissement du sapin s'est complètement interrompu. Donc, dans cette étude de cas, la dynamique du sapin est mieux décrite par un modèle de succession. La longue période ayant précédé l'établissement du sapin est liée possiblement à l'absence de semenciers alors que les sapinières mûres venaient tout juste d'être récoltées. Par contre, l'interruption observée depuis 1980 est plus difficilement explicable. Une «détérioration" progressive des lits de germination est possiblement en cause. Les divers travaux portant sur les successions après feu dans le domaine de la sapinière à bouleau blanc semblent indiquer que cette dynamique ne se limite pas à un seul cheminement successionnel. La régénération après feu serait largement influencée par le contexte local.

\section{Introduction}

Des peuplements bi-étagés feuillus/résineux se rencontrent fréquemment dans le domaine écoclimatique de la sapinière à bouleau blanc (Vézina 1966; Lafond et Ladouceur 1968; Cogbill 1984). Il s'agit de peuplements à deux étages où l'on distingue une strate arborescente feuillue dominée par le bouleau blanc (Betula papyrifera Marsh.) avec un sous-étage résineux dominé par le sapin baumier (Abies balsamea (L.) (Mill.) (Lafond et Ladouceur 1968).

Généralement, dans la forêt boréale du Québec, l'établissement d'un étage monospécifique de bouleau blanc suit un feu (Lortie 1979; Foster et King 1986) ayant complètement détruit le peuplement antérieur (Cogbill 1984). Les bétulaies blanches qui originent de coupe (Bélanger et al. 1989; Harvey et Bergeron 1989) ou d'une épidémie de tordeuses des bourgeons de l'épinette (Marchand 1991) ont plutôt un caractère mélangé. Après feu, le bouleau s'établirait rapidement par graines (Foster et King 1986) et par rejets de souche (Heinselman 1973). Les graines proviendraient de tiges survivantes ou de peuplements non brûlés attenants.

Département des sciences forestières, Faculté de foresterie et de géomatique, Université Laval, Sainte-Foy, Québec Canada G1K 7P4.

${ }^{1}$ Les résultats présentés proviennent du projet de fin d'études de Daniel Allard.
La description du processus d'établissement du sapin baumier est plus problématique. Deux hypothèses sont proposées dans la littérature. La première, qui se rattache au modèle classique de succession, suggère que le sapin s'établirait ultérieurement à la formation d'un couvert de bouleaux à partir de sapins semenciers entourant la zone incendiée (Vézina 1966; Lafond et Ladouceur 1968; Foster et King 1986). Le sapin est d'ailleurs considéré comme l'une des seules essences de remplacement en milieu boréal, une espèce qui envahirait graduellement un nombre important de types forestiers grâce à sa très grande tolérance à l'ombre (Dix et Swan 1971; Carleton et Maycock 1978). Suivant Foster et King (1986), l'établissement du sapin sous le couvert de bouleaux se ferait 40-50 ans après le feu; la détérioration du couvert feuillu s'amorcerait 75-100 ans après le feu, permettant alors une conversion progressive du peuplement en forêt résineuse ou mélangée.

Toutefois, selon Cogbill (1984), le modèle classique de succession, impliquant un recrutement graduel d'une nouvelle essence, s'appliquerait rarement dans le forêt boréale des Laurentides. Le processus successionnel en cause se rattacherait plutôt au modèle dynamique de la "composition floristique initiale" (Finegan 1984). Suivant ce modèle, les changements de composition du couvert arborescent seraient simplement l'expression de la croissance et de la longévité 
différentes des espèces qui se seraient établies simultanément peu après la perturbation. Il n'y aurait donc pas "succession". La très lente croissance juvénile du sapin comparativement au bouleau (Van Nostrand 1965; Logan 1965; 1969) expliquerait pourquoi le sapin demeure initialement opprimé en sous-étage du bouleau. Les observations de MacArthur (1964) dans des brûlis survenus après coupe en Gaspésie semblent corroborer ce modèle, les résineux s'établissant toutefois après un délai de quelques années alors que le bouleau s'installerait immédiatement après la perturbation. Bergeron et Dubuc (1989) expliquent également les processus de succession observés suite au feu dans la forêt boréale de l'Abitibi par les différences de longévité et les niveaux de tolérance à l'ombre des espèces établies initialement après la perturbation. Cependant, il faut noter que cette phase "initiale" d'établissement représenterait les 50 premières années après le passage du feu.

La prépondérance de l'un ou l'autre des deux modèles pourrait avoir des conséquences importantes au plan sylvicole. En effet, si le modèle successionnel classique s'appliquait, cela signifierait l'impossibilité d'accélérer la venue d'un peuplement résineux au stade de régénération à moins de faire appel au reboisement. Par contre, si le modèle de la composition initiale s'appliquait, il serait alors possible d'effectuer des coupes de nettoiement destinées à favoriser, dès le jeune âge, le développement des semis de sapins. Ceci accélérerait la succession en favorisant la venue d'une peuplement mélangé ayant une plus grande proportion de sapin dans la strate arborescente.

Cette recherche avait pour but de vérifier lequel des deux modèles pouvait s'appliquer aux peuplements de bouleaux biétagés situés dans la région écologique des Moyennes Laurentides de la Jacques-Cartier (Thibault 1985). Cette région boréale se distingue par la prédominance des sapinières en raison de la faible fréquence des incendies forestiers elle-même reliée à une forte pluviométrie (Jurdant et al. 1972). Une étude de cas a été réalisée dans un peuplement de 23 ha typique des bétulaies blanches monospécifiques de cette région.

\section{Cadre écologique}

Le peuplement étudié est situé à $80 \mathrm{~km}$ au nord de Québec $\left(47^{\circ} 18^{\prime} \mathrm{N}\right.$ et $\left.71^{\circ} 08^{\prime}\right)$, dans la région écologique des Moyennes Laurentides de la Jacques-Cartier, à l'intérieur du domaine écoclimatique de la sapinière à bouleau blanc (Thibault 1985). Le climat y est à la fois froid et humide avec une précipitation moyenne annuelle élevée (1 $430 \mathrm{~mm})$ et une température moyenne annuelle de $0,3^{\circ} \mathrm{C}$ (station météorologique de la Forêt Montmorency). Le peuplement sélectionné avait une superficie initiale de 23 ha. De façon générale, aucun point dans ce peuplement n'était distant de plus de $150 \mathrm{~m}$ des sapinières avoisinantes ou des îlots résineux localisés dans la bétulaie. Des coupes, rapportées en 1935-36 par le concessionnaire forestier de l'époque (Côté 1946) permettent de supposer que le peuplement précédent présentait une forte proportion résineuse. L'incendie forestier a probablement pris naissance en 1936 au cours des travaux de récolte dans ce secteur.

Le présent peuplement peut être classé dans la sousassociation de la bétulaie blanche à dryoptéride (Gerardin 1977) et crôit sur un till sablo-loameux épais bien drainé. La végétation du sous-bois est dominée par le sapin, Dryopteris spinulosa (O.F. Muell) Watt., Clintonia borealis (Ait.)
Raf. et Oxalis montana Raf. La couverture muscinale y est très peu développée.

\section{Méthode}

La strate arborescente de bouleau a été échantillonnée suivant un dispositif systématique en 1986 à l'aide de 34 parcelles à rayon variable dans lesquelles les tiges ont été récoltées (Meek 1992) pour fin d'analyse de la tige. Pour échantillonner la strate arbustive de sapin, neuf parcelles de 200 mètres carrés ont été distribuées de façon systématique en 1990 suivant un gradient de distance par rapport aux sapinières attenantes. Une distance maximale de $150 \mathrm{~m}$ séparait les placettes d'un couvert arborescent de sapin et donc d'une source de semences résineuses.

Chaque parcelle de $200 \mathrm{~m}^{2}$ a fait l'objet d'un dénombrement complet permettant l'estimation de la densité. Dix sapins du sous-étage résineux ont été récoltés de façon aléatoire dans chacune des placettes pour un total de 90 échantillons. Ces derniers ont été utilisés pour établir la structure d'âge de la population de sapins. L'âge des sapins baumiers fut établi en laboratoire par dénombrement des anneaux de croissance au collet à l'aide d'un microscope binoculaire.

\section{Résultats}

L'étage supérieur du peuplement se compose à $97 \%$ de bouleau blanc; le cerisier de Pennsylvanie (Prunus pensylvanica L.f.) et le sapin baumier complètent cette cohorte. Cette strate dominante se caractérisait en 1986 par une densité moyenne à l'hectare de 3307 (écart-type $=1195 / \mathrm{ha}$ ) tiges de $1 \mathrm{~cm}$ et plus au dhp. Le diamètre moyen était de $9,9 \mathrm{~cm}$, la surface terrière de $27,4 \mathrm{~m}^{2} /$ ha et le volume total de $134 \mathrm{~m}^{3} / \mathrm{ha}$. La hauteur du couvert était de 12,1 m. La plupart des bouleaux se seraient établis moins de six ans après le feu (figure 1). Très peu de rejets de souches ont été observés. La présence de souches de sapin brûlées semble confirmer le présence d'une sapinière antérieurement au feu. Les semences proviendraient des secteurs de coupe adjacents où le bouleau était laissé sur pied. L'âge médian en 1990 était de 52 ans.

La sapin compose l'essentiel de l'étage inférieur. Cet étage avait une hauteur moyenne de deux mètres, une hauteur relativement élevée pour une régénération préétablie de sapin (Côté et Bélanger 1991). La densité était de 2072 tiges/ha (écart-type = 1 978/ha).

Cette régénération résineuse est beaucoup moins dense mais beaucoup plus haute comparativement à la régénération préétablie de sapin retrouvée dans les sapinières d'âge comparable où les densités dépassent 10000 semis/ha mais où la plupart des semis ont moins de $30 \mathrm{~cm}$ de hauteur (Côté et Bélanger 1991). L'âge médian était de 21 ans en 1990, soit deux fois plus jeune que le bouleau.

L'établissement du sapin baumier semble avoir suivi un patron particulier (figure 1) qui comprend cinq phases:

-1936 à 1951 (16 ans) :

aucun établissement du sapin

- 1952 à 1963 (12 ans) :

établissement sporadique de sapin

- 1964 à 1976 (13 ans) :

établissement abondant de sapin

- 1977 à 1980 (4 ans) :

établissement sporadique de sapin

-1981 à 1990 (10 ans) :

aucun établissement du sapin. 
Bouleau blanc $\square$ Sapin baumler

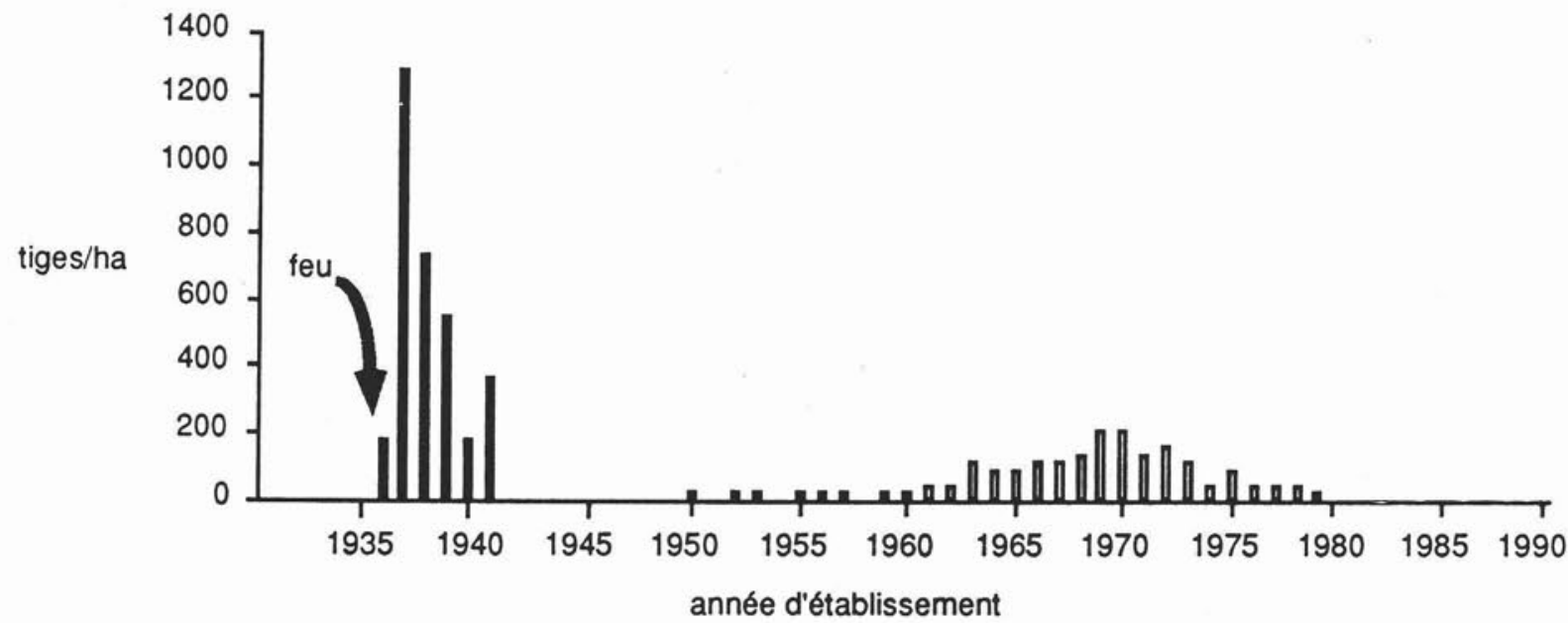

Figure 1. Structure d'âge mesurée en 1990 des arbres d'un peuplement bi-étagé de bouleau blanc avec sapin baumier.

Ce patron d'établissement du sapin donne une structure d'âge des sapins qui se rapproche d'une distribution normale. Le pic d'établissement en situe en 1968-70, soit 33 ans après le feu alors que les bouleaux se sont surtout établis deux à quatre ans après le feu.

L'observation d'un certain dépérissement du bouleau blanc indiquerait que celui-ci commence maintenant à céder sa place au sapin. Suite aux trouées dues à la sénescence du bouleau, les sapins devraient amorcer leur croissance en hauteur. On peut donc s'attendre à moyen terme à ce que ce peuplement feuillu bi-étagé se développe en un peuplement mélangé composé de bouleaux et de plus petits sapins. Ce peuplement correspondrait à la bétulaie blanche à sapin et épinette blanche décrite par Lafond et Ladouceur (1968). Ces derniers interprétaient à l'époque ce type forestier comme étant le stade final (climax) dans le domaine de la sapinière à bouleau blanc.

\section{Discussion}

La strate arborescente de bouleau blanc observée dans cette étude a une structure analogue à celle rapportée par Cogbill (1984) pour les bétulaies blanches régénérées après un feu. Très équien, l'étage monospécifique de bouleau s'est établi immédiatement après le feu pendant une période relativement courte. La période d'établissement a été de six ans. Ceci se compare bien à la période d'établissement de 15 ans rapportée par Foster et King (1986) dans le cas de bétulaies régénérées après feu et à la période de 10 ans rapportée par MacArthur (1964) dans le cas de bétulaies régénérées après une coupe suivie d'un feu. Le fait que le feu ait suivi une coupe ne semble pas affecter la dynamique du bouleau blanc.

Sachant que la dispersion de la majorité des graines de bouleau blanc ne dépasse pas beaucoup $100 \mathrm{~m}$ (Marquis 1969, Archibold 1980), les semenciers étaient probablement constitués par les bouleaux laissés sur pied dans les coupes attenantes au secteur incendié.

Pour ce qui est du sapin baumier, l'ensemble des individus étudiés s'est établi 10 à 40 ans après la population de bouleau avec un pic d'établissement 33 ans après le feu. Dans ce cas, la dynamique végétale s'apparente donc au modèle traditionnel de la succession. Même en utilisant des classes d'âge de 20 ans, le bouleau et le sapin ne font pas partie la même cohorte. La structure du peuplement étudié s'apparente à celle décrite par Foster et King (1986) bien que dans leur cas l'établissement prolifique du sapin était 40-50 ans après le feu. Cogbill (1984) note également que le pic d'établissement du sapin est 10-20 ans après celui du bouleau mais suggère, qu'ils font toujours partie de la même cohorte.

MacArthur (1964) a également étudié en Gaspésie la dynamique d'établissement de brûlis qui avaient comme origine une coupe suivie d'un feu. Or ce dernier a observé un patron d'établissement des sapins analogue à celui observé dans cette étude. Dans ce cas toutefois, le patron était plus court, l'établissement du sapin commençant 4-6 ans après le feu, atteignant un pic 9 à 10 ans après le feu pour diminuer jusqu'à devenir nul 13 à 18 ans après le feu. La présence de semenciers mûrs attenant le brûlis pourrait expliquer l'établissement plus rapide des résineux dans ce cas.

L'interprétation du patron d'établissement du sapin observé dans notre étude doit tenir compte du fait que le feu de 1936 s'est produit dans un secteur qui venait de subir une coupe sur de grandes superficies. Ceci implique qu'immédiatement après le feu, il devait y avoir relativement peu de semenciers de sapin dans les environs. Par contre, comme le bouleau n'était pas récolté, une réserve de semenciers était présente. Ainsi, la première période où il n'y a eu aucun établissement de sapin pourrait possiblement s'expliquer par l'absence de semenciers de sapin. D'ailleurs, les deux secondes phases du patron d'établissement du sapin observé correspondent parfaitement au développement normal de la production semencière du sapin. Celui-ci commence à produire des graines vers l'âge de 15 ans mais une production semencière régulière et abondante ne s'amorce que vers l'âge de 30 ans (Fowells 1965). Quant à la seconde période 
d'établissement sporadique de sapin entre 1977-1980, elle pourrait s'expliquer par l'épidémie de tordeuses des bourgeons de l'épinette amorcée en 1975 (Blais 1985); il est reconnu qu'une telle épidémie affecte directement la production de semences par le sapin (Powell 1973; Schooley 1978).

C'est la dernière phase, où il y a absence complète d'établissement de sapin entre 1981 et 1990, qui pose des difficultés d'interprétation. Trois hypothèses peuvent possiblement expliquer cette absence de régénération de sapin: l'absence d'une production semencière, des difficultés de dispersion des graines et des problèmes de germination et de survie pour les jeunes semis.

L'absence de semences due à l'épidémie de tordeuses des bourgeons de l'épinette semble être peu probable. En effet, deux très bonnes années semencières ont été observées dans le secteur en 1984 et 1988 (Côté et Bélanger 1991). Elles auraient dû permettre à tout le moins l'établissement d'un certain nombre de semis tout comme lors de la première phase de l'épidémie.

Il se peut que la croissance de la strate arborescente de bouleau ait créé une barrière physique limitant la dissémination des semences à partir des sapinières avoisinantes. À première vue, toutefois, cette hypothèse reste peu probable. Les différences de hauteur des bouleaux entre 1975 et 1980 semblent peu importantes pour expliquer une barrière possible à la dispersion des graines.

Par contre, l'hypothèse de MacArthur (1964) selon quoi le cycle de régénération du sapin après feu serait lié à l'évolution des milieux de croissance et/ou de la végétation du sous-bois semble être plus plausible. Koroleff (1954), Place (1955), Gregory (1966), Côté et Bélanger (1991) ont déjà noté en zone boréale que les litières de feuilles semblent entraver la germination et la survie des résineux. L'évolution de l'humus sous la bétulaie et notamment l'accroissement en épaisseur de l'horizon fibrique depuis le feu pourraient être en cause en agissant comme barrière physique aux racines des jeunes semis. Des effets allélopathiques ou autres associés à l'évolution de la végétation du sous-bois pourraient aussi jouer un rôle (Ahlgren et Ahlgren 1981). Enfin, Gregory (1966) a bien mis en évidence les effets mécaniques de la chute annuelle des feuilles de bouleau qui écrasent et étouffent la régénération résineuse.

\section{Conclusion}

Les divers travaux portant sur les successions après feu dans le domaine de la sapinière à bouleau blanc semblent à première vue contradictoires. Alors que les observations de MacArthur (1964) en Gaspésie suivent le modèle dynamique de la composition initiale, celles réalisées dans cette étude suivent plutôt un modèle successionnel classique. Il semble donc difficile de généraliser un modèle dynamique contrairement à ce que suggèrent les schémas utilisés pour décrire les séries évolutives. Le contexte local (dimension de la perturbation, présence de semenciers, etc.) aurait une influence déterminante sur l'évolution de ces peuplements après feu.

\section{Références}

Ahlgren, C.E. et I.F. Ahlgren. 1981. Some effects of different forest litters on seed germination and growth. Can. J. For. Res. 11: 710-714.
Archibold, O.W. 1980. Seed input into a postfire forest site in northern Saskatchewan. Can. J. For. Res. 10: 129-134. Bélanger, L., P. Bouliane et M. Pineau. 1989. Problématique de la sapinière boréale de seconde venue. Dans Colloque sur la sapinière boréale, Forêt Montmorency, Québec. Ordre des ingénieurs forestiers du Québec.

Bergeron, Y. et M. Dubuc. 1989. Succession in the southern part of the Canadian boreal forest. Vegetation 79: 51-63.

Blais, J.R. 1985. Répercussion des invasions de la tordeuse des bourgeons de l'épinette sur le sapin baumier et l'épinette blanche dans la réserve des Laurentides (rapport d'étape). Rapp. Inf. LAU-X-68F. Cent. Rech. For. Laurentides, Québec. $16 \mathrm{p}$.

Carleton, T.J. et P.F. Maycock. 1978. Dynamics of the boreal forest south of James Bay. Can. J. Bot. 56: 1157-1173. Cogbill, C.V. 1984. Dynamics of the boreal forest of the Laurentian Highlands, Canada. Can. J. For. Res. 15: 252-261.

Côté, P.H. 1946. Plan général d'aménagement: concessions forestières de la rivière Montmorency, P.Q. Département du bois, Anglo-Canadian Pulp and Paper Mills, Limited, Québec, $611 \mathrm{p}$.

Côté, S. et L. Bélanger. 1991. Caractérisation de la régénération prétablie dans les sapinières boréales en fonction de leur situation écologique. Can. J. For. Res. 21: 1779-1795. Dix, R.L. et J.M.A. Swan. 1971. The roles of disturbance and succession in upland forest at Candle Lake, Saskatchewan. Can. J. Bot. 49: 657-676.

Finegan, B. 1984. Forest succession. Nature 312: 109-114. Foster, D.R. et G.A. King. 1986. Vegetation pattern and diversity in S.E. Labrador, Canada: Betula papyrifera (birch) forest development in relation to fire history and physiography. J. Ecol. 74: 465-483.

Fowells, H.A. 1965. Sylvics of forest trees of the United States. Agriculture Handbook no 271. USDA For. Serv., Washington, DC. $762 \mathrm{pp}$

Gagnon, R. 1989. Maintien après feu de limites abruptes entre des peuplements d'épinette noire (Picea mariana) et des formations de feuillus intolérants (Populus tremuloides et Betula papyrifera) dans la région du Saguenay-Lac-SaintJean (Québec). Nat. can. 116: 117-124.

Gerardin, V. 1977. An integrated approach to the determination of ecological groups in vegetation studies. Thèse de doctorat, Univ. Connecticut. 237 p.

Gregory, R.A. 1966. The effect of leaf litter upon establishment of white spruce beneath paper birch. For. Chron. 42: 251-255.

Harvey, B.D. et Y. Bergeron. 1989. Site patterns of natural regeneration following clear-cutting in northwestern Quebec. Can. J. For. Res. 19: 1458-1469.

Heinselman, M.L. 1973. Fire in the virgin forest of the Boundary Waters Canoe Areas, Minnesota. Quaternary Research 3: 329-382.

Jurdant, M., J. Beaubien, J.L. Bélair, J.C. Dionne et V. Gerardin. 1972. Carte écologique de la région du Saguenay-Lac-St-Jean. Notice explicative. Vol. I - L'environnement et ses ressources: identification, analyse et évaluation. Rapport d'information Q-F-X-31. Centre de foresterie des Laurentides. Env. Canada, Québec. 93 p.

Koroleff, A. 1954. Leaf litter as a killer. J. For. 2: 178-182. Lafond, A. et G. Ladouceur. 1968. Description des groupe- 
ments forestiers du Québec. Ministère des Terres et Forêts, Québec. 726 p.

Logan, K.T. 1965. Growth of tree seedlings as affected by light intensity. I. White birch, yellow birch, sugar maple and silver maple. Dept. of forestry publication no 1121, Forest Research Branch, Canada. 16 p.

Logan, K.T. 1969. Growth of tree seedlings as affected by light intensity IV. Black spruce, white spruce, balsam fir and eastern white cedar. Dept. of Fish. and For. Serv. publication no 1256, Can. For. Serv., Canada. 13 p.

Lortie, M. 1979. Arbres, forêts et perturbations naturelles au Québec. Presses de l'Université Laval, Québec. 172 p. MacArthur, J.D. 1964. A study of regeneration after fire in the Gaspé region. Dept. of forestry publication no. 1074, Forest Research Branch, Canada. 20 p.

Marchand, S. 1991. Dynamique de la régénération naturelle de jeunes sapinières boréales du centre du Québec. Mémoire de maîtrise, Faculté de foresterie et de géomatique, Université Laval, Québec. 79 p.

Marquis, P.A. 1969. Silvical requirements of natural birch regeneration. Dans Birch Symposium Proceedings, USDA
Northeast Forest Research Paper NE-146.

Meek, P. 1992. Caractéristiques allométriques du bouleau blanc en zone boréale. Mémoire de maîtrise, Faculté de foresterie et de géomatique, Université Laval, Québec.

Place, I.C.M. 1955. The influence of seedbed conditions on the regeneration of spruce and balsam fir. Can. Dep. North. Aff. Nat. Resour. For. Branch. Bull. 177.

Powell, G.R. 1973. The spruce budworm and megasporangiate strobili of balsam fir. Can. J. For. Res. 3: 424-429. Schooley, H.O. 1978. Effects of spruce budworm on cone production of balsam fir. For. Chron. 54: 298-301.

Thibault, M. 1985. Les régions écologiques de Québec méridional, deuxième approximation. Ministère de l'Énergie et des Ressources, Québec (carte).

Van Nostrand, R.S. 1965. Results of experimental seeding of balsam fir on a recent burn. Dept. of forestry publication no. 1103, Forest Research Branch, Canada. 11 p.

Vézina, P.-E. 1966. Le traitement de peuplements mélangés bi-étagés sapin/bouleau. Dans Coté, M., Plan général d'aménagement de la Forêt Montmorency de l'Université Laval. Presses de l'Université Laval, Québec. pp. 136-139.
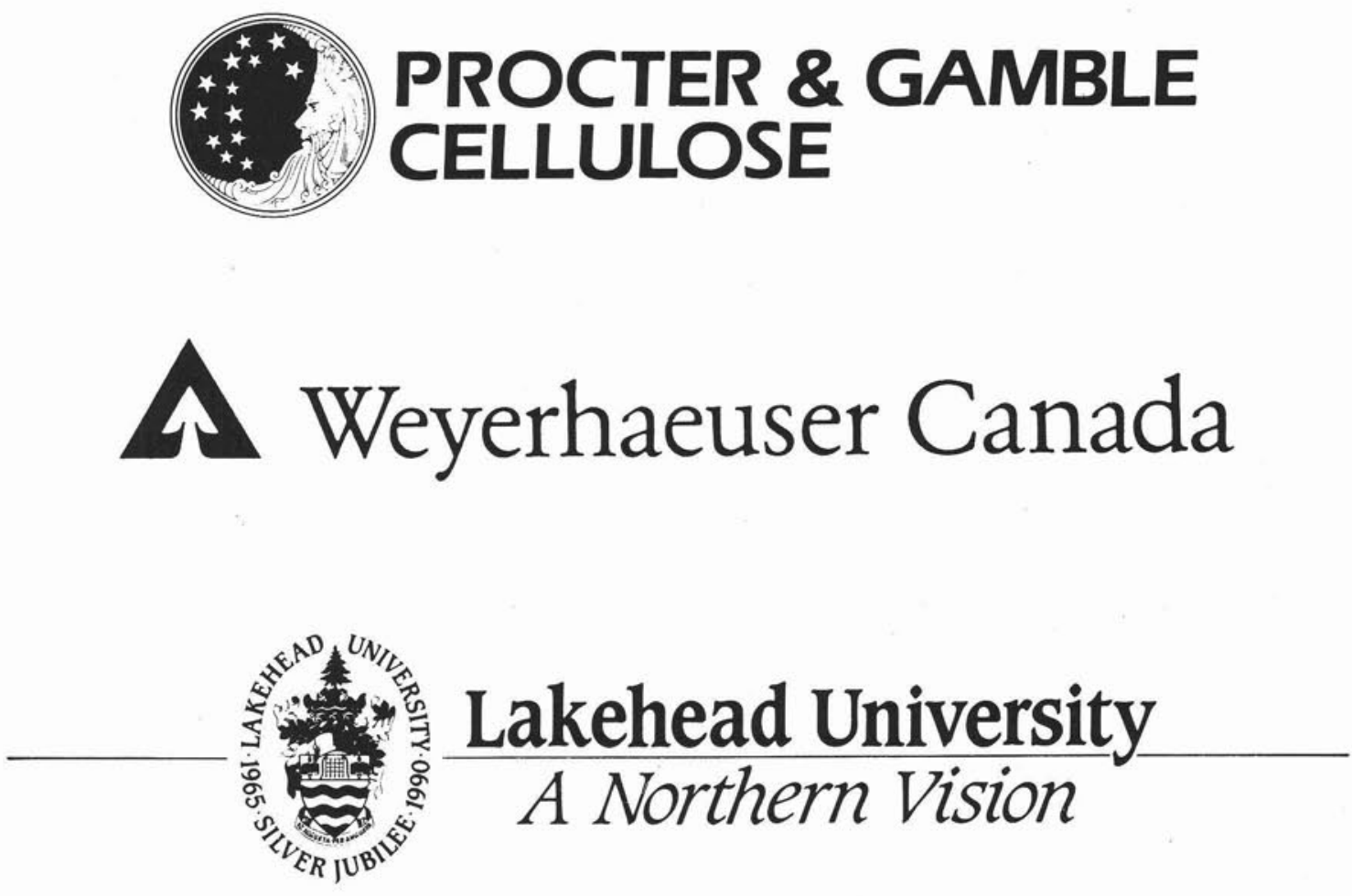

CIF/IFC Corporate Sustaining Members 